

\section{Uma experiência de limiar: sobre a medialidade dos jogos (de computador)}

\author{
A threshold-experience: about \\ mediality on computer games
}

\section{Resumo}

O presente texto realiza um breve estudo conceitual sobre o jogo e o lúdico. A partir da exposição das estruturas mediais e ontológicas do jogo, este trabalho argumenta sobre a sua particularidade como uma experiência de limiar. Por fim, por meio de uma caracterização das especificidades dos jogos de computador, levanta-se a discussão sobre um conflito conceitual entre jogo e comunicação.

\section{Palavras-chave}

Teorias dos jogos; teorias da comunicação; teorias dos media; filosofia dos media.

\section{Abstract}

This paper carries out a brief conceptual study on game and play. From the enunciation of play's medial and ontological structures, this work argues about game's own particularity as a thresholdexperience. Finally, through a characterization of the specificities of computer games, a discussion about a conceptual conflict between game and communication is outlined.

\section{Keywords}

Game studies; communication theory; media theory; media philosophy.

\section{DOSSIÊ}


Este breve ensaio trata de uma ilusão. Mas ilusão não se refere aqui apenas a alguma coisa que pareça algo que de fato não é. Do latim illudus, essa palavra contém em si o brincar, o jogar, o divertir-se, o lúdico (ludus). Por isso que em sua origem latina, a palavra ilusão também significa brincar com, jogar com (Hoad, 1996). Esses dois elementos da ilusão, o "como se" e o "com" como algo que põe alguma coisa em jogo, são apreendidos neste texto como uma espécie de motor do lúdico. Em outras palavras, o conceito de ilusão aponta para uma estrutura que possibilitaria o jogo. A descrição dessa estrutura - que por compor mundos dentro do mundo (ao mesmo tempo em que se faz esquecer) se apresenta como medialidade - constitui a primeira parte deste trabalho.

Em um segundo momento, mais especulativo, a discussão teórica sobre jogo deságua na problemática da comunicação em meios digitais. A principal hipótese deste trabalho é que a função medial do jogo é essencialmente diversa da relação comunicacional, cujo fundamento encontra-se em uma experiência da alteridade. Como será discutido adiante, o lúdico é sempre uma experiência de limiar. Essa característica é exposta ao se discutir máquinas digitais a partir de suas formas relacionais/comunicativas, já que o lúdico, ou o modo de ser do jogo, é radicalizado em espaços digitais através da obediência à sua lógica matemática imanente.

\section{Medialidade do jogo}

Em um sentido mais geral, todo ato lúdico é uma atividade livre e divertida que escapa à esfera produtiva e mandatória do trabalho. Quando o lúdico está estruturado a partir de regras e metas, ele constitui um jogo. O lúdico, portanto, seria o fundamento de qualquer jogo: é o que dá sua lógica, sua medialidade, seu modo de ser. Além disso, o lúdico é uma forma de experiência que transcende o espaço do jogo. Ele constitui um modo de relação o qual eu chamo aqui de "limiar": um espaço "entre" cujo principal atributo é criar uma espacialidade segura, produzir o intervalo do "como se", no qual a possibilidade de experimentação deve ser garantida. A caracterização desse modo de relação é o objeto deste tópico.

O filósofo alemão Hans-Georg Gadamer, em sua obra Verdade e Método (originalmente publicada em 1960), reflete sobre o modo de ser do jogo para salientar suas similaridades com o ser da obra de arte: ambos encontram-se fechados em um mundo inteiramente autônomo (embora sua auto-apresentação possa ser apresentada a outra pessoa) e eles se renovam o tempo todo (como um eterno movimento de ida e volta). Para Gadamer, o jogo e a arte são experienciados como realidades que superam a realidade do jogador/espectador.

Com o intuito de evitar definições culturais, sociais e psicológicas do jogo centradas nos jogadores e nas suas ações, Gadamer ressalta a função medial do jogo. Lendo a obra Homo Ludens, um marco para os game studies e publicado em 1944 pelo antropólogo holandês Johan Huizinga - que concebia o lúdico como uma atividade livre que ocorre em um espaço sagrado, i.e. um espaço separado da vida ordinária (1980, p. 14) Gadamer define o jogo, acima de tudo, como sendo independente da consciência do jogador (1975, p. 108 [97/98]).

A primazia do jogo em relação aos jogadores pode ser entendida como uma forma de expor suas estruturas de outridade. $E$ isso implica pelo menos dois fatos: 1. para que um jogo "seja", é sempre necessário um "ou- tro", de tal maneira que sempre haverá uma resposta e, portanto, um "acionamento" da estrutura de jogo (Gadamer, 1975, p. 111 [100/101]); 2. jogadores não jogam, mas são jogados - eles estão sujeitos ao jogo: "Todo jogar é ser-jogado" (Gadamer, 1975, p. 112 [101/102]).

O primeiro fato garante o eterno movimento do jogo. É o que produz tensão, incerteza e descoberta. O segundo atesta que todo jogar é um jogar-com e, consequentemente, um lidar com uma outridade que não pode ser, de início, inteiramente concebida ou apreendida. O fato de que o "jogo é o sujeito do jogar" já seria responsável por provocar uma experiência de estranheza, de outridade.

Entretanto, a função medial do jogo é diferente daquela da arte. O filósofo francês Roger Caillois elaborou uma classificação do lúdico e do jogo a partir do trabalho de Huizinga. Em seu livro Men, Play and Games (publicado em 1958), ele caracteriza o lúdico como livre, separado da vida ordinária, incerto e improdutivo (assim como a obra de arte), mas acima de tudo como algo regulado por regras e estruturado como faz-de-conta (2001, p. 9-10).

Essas duas últimas características constituem o fundamento do jogo: as regras, os ordenamentos e o "como-se" prescrevem e determinam o espaço sagrado do jogo. Por sua vez, as obras de arte podem reinventar suas regras infinitamente até que nenhuma regra ou regularidade possa ser mais aplicada - e ainda assim elas continuariam a ser consideradas obras de arte. Por outro lado, o jogo deve permanecer em suas regras, pois elas lhe são imanentes.

As regras e o faz-de-conta revelam a medialidade do jogo. Isso já foi percebido por Huizinga, que define aquilo que é característico do lúdico como "a consciên- 
cia, mesmo que latente, de 'apenas fingir'"' (1980, p. 22). No entanto, foi o antropólogo inglês Gregory Bateson em seu artigo A Theory of Play and Fantasy, de 1954, que desenvolveu os enredamentos ontológicos e mediais do jogo ao aproximá-lo da noção de sonho lúcido um limiar entre duas realidades incompatíveis mas coexistentes.

\section{In-lusio}

Para Bateson, todo jogo se estabelece a partir da metacomunicação "isto é um jogo/isto é uma brincadeira". Como ele esclarece, a declaração "isto é um jogo" pode ser compreendida da seguinte maneira: "Essas ações com as quais nós estamos comprometidos agora não denotam aquilo que essas ações normalmente denotariam neste lugar" (1972, p. 184). Isso corresponde, em seu significado, ao de uma ilusão consentida. "Isto é um jogo" opera algo que é ligeiramente (mas essencialmente) diferente da função simbólica da preposição "como" - ou seja, daquilo que indica que algo está para outra coisa. Essa metacomunicação designa antes uma alusão (literalmente um "brincar, jogar com"), uma evocação de uma aparência, mas não da coisa ou do ato em si. Quando as crianças brincam de luta, um soco deve parecer um soco, mas, obviamente, ele não deve machucar como um de verdade. Isso é próximo à ideia de simulação. Assim, o modo de ser do jogo — ou seja, o lúdico - é distinto não pela estrutura simbólica do "como", mas sim pela estrutura performativa do "como se". Como Caillois já apontou, isso "implica as regras que estão por trás de todos os jogos e atividades lúdicas. Isso cria ficção, ilusões (in-lusio)" (2001, p. 8).
A medialidade do jogo com-põe não apenas uma outra realidade, mas também instaura um limiar entre pares de camadas incompatíveis, tais como realidade e ficção, jogadores e espectadores, o discursivo e o performativo. Esta característica única foi exemplarmente caracterizada por Bateson como a equação e o discernimento simultâneos entre mapa e território.

É por esse motivo que a metacomunicação "isto é um jogo" constitui um "enquadramento" que assevera a coexistência de instâncias incompatíveis. A partir da noção de quadro, Bateson destaca duas principais peculiaridades do jogo: "(a) que as mensagens ou sinais trocados durante o jogo são, em certo sentido, falsos ou não significam o que querem dizer; e (b) que o que é denotado por esses sinais é inexistente" (1972, p. 186) Essas particularidades também são partilhadas pela arte, pelo ritual, pela magia e pela religião. Mas apenas "no jogo", através de sua constante metacomunicação, é que o enquadramento é sempre experienciado como enquadramento.

A superação desse limiar implica o fim do jogo. A simultaneidade de um "dentro" e de um "fora" do jogo é um vestígio tanto de sua extrema fragilidade quanto de sua extrema liberdade. Jogar é sempre um ato voluntário: "Também não há dúvida de que o lúdico deve ser definido como uma atividade gratuita e voluntária, uma fonte de alegria e diversão. Um jogo no qual alguém fosse forçado a jogar imediatamente deixaria de ser um jogo" (Caillois, 2001, p. 6). Por isso, o enquadramento "isto é um jogo" não possui apenas implicações ontológicas, mas também éticas. $E$ a impossibilidade de transgredir esse enquadramento reassegura a imanência da sua ilusão.

\section{Ética enquadrada}

Quem é o "outro" no jogo? Um membro da equipe? Um adversário? O próprio jogo? Como as ações e os costumes no jogo podem ser avaliados? Para responder a essas perguntas, é preciso recorrer à estrutura "isto é um jogo", pois a promessa desta metacomunicação garante não somente a sua existência, mas sua ética. Dessa forma, a outridade do/no jogo é particularmente normativa: ela é dada pelas regras e/ou pelo faz-de-conta. Consequentemente, as questões éticas de jogo estão atadas às suas diretrizes. Um comportamento ético é o fair play, o jogo justo. É ser mutuamente justo. Se a ordem do jogo é transgredida, esta ética normativa colapsa: o bem e o mal, o certo e o errado, a virtude e o vício, a justiça e o crime, todos eles apenas existem na disposição intrínseca do jogo.

Esta ética normativa e imanente do lúdico implica que um jogo pode ser sério, mas sua seriedade não denota responsabilidade, dever ou mesmo uma tarefa isso também é o que distingue o lúdico de um ritual e de uma cerimônia. Esse aspecto já havia sido reconhecido por Huizinga (1980, p. 8): “O jogo é supérfluo. A necessidade por ele é urgente na medida em que a sua satisfação o torna uma necessidade. $O$ jogo pode ser diferido ou suspenso a qualquer momento. Ele nunca é imposto por necessidade física ou dever moral".

Logo, o supérfluo é, por um lado, essencial para a liberdade e incerteza do jogo - o que asseguraria a tensão e o desejo de se manter jogando. Entretanto, a liberdade de escolha é uma reação à estrutura de jogo. A liberdade de jogo demanda uma resposta. Mas o jogo só pode ser livre apenas dentro dos limites dispostos pelas suas regras. Desta forma, a liberdade de escolha é ao mesmo tempo canalizada, potencializada 
e comprometida pelas ordens e ordenamentos do jogo.

Por outro lado, o supérfluo do jogo é fundamental para a composição de um espaço seguro e reversível é por isso que ele se torna o lugar de experimentação "por excelência". Essa capacidade de criar mundos dentro do mundo institui a relevância pedagógica do jogo e o aproxima das experiências místicas e estéticas. Ela possibilita uma suspensão temporária da vida ordinária e abre um limiar no qual o participante se torna "diferente e faz coisas diferentemente" (Huizinga, 1980, p. 12)

Naturalmente, o tempo e o espaço alterados do jogo são essencialmente simplificados: "As leis confusas e intrincadas da vida comum são substituídas, neste espaço fixo e por esse tempo determinado, por regras precisas, arbitrárias e irreconhecíveis que devem ser aceitas como tal e que regem o jogar correto do jogo" (Caillois, 2001, p. 7).

Além disso, essa outra espacialidade e essa outra temporalidade instauradas pelo jogo são "improdutivas": mesmo quando o jogo afeta diretamente a realidade circundante - como, por exemplo, nos jogos de azar - o que é apostado não é um produto do próprio jogo. Em contraste, o que é produzido durante o jogo não pode ter a mesma função fora dele. Portanto, o excesso e o desperdício são atributos do jogo. Como Caillois (2001, p. 5) explica, uma "característica do jogo, de fato, é que ele não cria riqueza ou bens, diferindo assim do trabalho ou da arte. Ao final do jogo, tudo pode e deve recomeçar no mesmo ponto".

O movimento de ida e volta do jogo também instaura a possibilidade de quebrar e transformar as suas regras. Embora o outro-no-jogo já esteja dado pela sua lógica interna, a sua condição de limiar atesta sua abertura persistente a uma virada ou quebra iminente. Isso significa também que a alteridade do jogo não é jogável, pois ela escapa à sua lógica. No mundo estabelecido durante o jogo reina uma alteridade enquadrada: uma experiência de estranheza diante de um novo desafio e em seguida, sua consequente superação ou adaptação.

É por isso que não seja surpresa alguma que as teorias matemáticas dos jogos (Von Neumann; Morgenstern, 1944) tentaram calcular a incerteza do jogo, ou seja, suas situações, movimentos, escolhas, em suma, sua lógica de decisão. Após a Segunda Guerra Mundial, a semelhança entre jogo e simulação encontrou seu apogeu através da capacidade de cálculo das máquinas digitais. Os jogos de computador inauguraram uma era única onde os processos de enquadramento do jogo e sua consequente proximidade com a lógica matemática foram radicalizados. Eles podem ser considerados "o" meio de expressão da era digital porque os jogos de computador sintetizam seus conceitos-chave, a saber, interação, interconexão, controle e decisão, e aprofundam seus potenciais éticos e estéticos. Além disso, jogos de computador podem ser aplicados como uma figura para descrever uma maneira ou um modelo medial predominante de lidar com as tecnologias digitais. Portanto, a discussão sobre jogos de computador pode ser útil para expor a problemática da alteridade e da comunicação através dos meios digitais.

\section{Ilusão digital}

Já na sua época, Roger Caillois (2001, p. 174) havia criticado as teorias matemáticas dos jogos porque elas destroem a razão de ser do lúdico, isto é, a contingência, enquanto tentam determinar matematicamente todas as situações e movimentos possíveis de um jogo; eles o transformam em informações, ou seja, em uma tomada de decisão dentro de um certo campo de possibilidades. Mas o que acontece quando o cálculo se torna o fundamento do próprio jogo?

Para além de todos os principais atributos do jogo - liberdade (participação não obrigatória), autonomia (tempo e espaço próprios), dúvida ou tensão (que requer participação ativa dos jogadores) e regras - um jogo de computador também é baseado em matemática cibernética. Isso significa dizer que na base de qualquer jogo digital encontra-se um modelo de decisão. De acordo com o que o filósofo alemão Dieter Mersch apresentou através da sua desconstrução do pensamento cibernético no livro Ordo ab Chao: Order from Noise (2014), a formalização matemática é o núcleo de todos os dispositivos computacionais.

Assim, o aparato tecnológico que possibilita um jogo de computador é fundamentado por conceitos cibernéticos, a saber, o controle, a autorregulação, a recursividade, a autorreflexividade e a autorreferencialidade. Essa peculiaridade já foi ressaltada por muitos pesquisadores de jogos de computador que tentaram definir as particularidades do medium videogame (Beil, 2013; Distelmeyer, Hanke, Mersch, 2008; Günzel, 2012; Pias, 2002; Wolf, 2010). A matemática computacional é a razão pela qual a expressão "jogos de computador" ou "jogos digitais" seria mais apropriada do que o termo videogames, uma vez que eles estão sempre sujeitos à lógica computacional.

Em adição à sua intrínseca lógica matemática, do ponto de vista da medialidade, existem pelo menos três outras diferenças entre jogos digitais e não-digitais: a incapacidade de criar contingência; a interação e seu consequente efeito de imersão como o elemento genuíno do jogar digital para revelar os outros espaços 
do jogo; e a ignorância sobre a totalidade das regras, bem como a impossibilidade de sua subversão.

No que diz respeito à primeira diferença, o teórico da mídia Markus Rautzenberg (2015) defende que o jogo digital só pode produzir uma "incerteza emoldurada". Isso significa que os jogos de computador articulam um paradoxo: simulam a incerteza (normalmente através da aleatoriedade), mas não podem criar contingência, pois ela estará sempre inscrita no código do jogo. Como Rautzenberg $(2015$, p. 68) explica, os jogos de computador "são baseados em seus níveis técnicos em computadores que, na forma da combinatória atualmente dominante baseada na arquitetura de Von Neumann e nas máquinas de Turin, não são capazes de gerar aleatoriedade real e entropia".

Por outro lado, o poder imersivo dos jogos de computador consiste exatamente em criar um mundo autossuficiente onde as ações do jogador sejam realizadas com o desconhecimento do sistema total de regras, o que cria uma ilusão de soberania sobre o campo de ação. Além disso, para sustentar o sentimento da imersão, existem dois modos de jogo que são complementares, mas não coincidentes: o performativo e a narrativo. Essa categorização foi desenvolvida por Dieter Mersch (2008, p. 33) em seu estudo sobre a medialidade e a lógica dos jogos digitais. "Grosso modo", essas duas formas de jogo descrevem o fato de que quando um jogo de computador é jogado, não é narrado; e quando é narrado, não é jogado. Nós poderíamos assumir essa divisão quase que literalmente, porque é bastante comum que os jogos de computador modernos tenham várias cenas de corte fundamentais para a trama do jogo, às quais o jogador apenas assiste.

No entanto, essas categorias também podem ser entendidas como modos de experimentar um jogo de computador, especialmente porque existem jogos em que as cenas de corte foram sutilmente integradas à ação, como por exemplo The Last of Us (Naughty Dog, 2013). A performatividade e a narratividade podem ser formas de experiência da mesma forma que categorias como o digital e o analógico são formas de leitura. Em seu livro Languages of Art: An Approach to a Theory of Symbols (1968), o filósofo americano Nelson Goodman fornece uma distinção básica entre um esquema de representação digital e analógico: o primeiro é diferenciado ou discreto, enquanto o último é contínuo ou denso. A filósofa alemã Simone Mahrenholz (2003) aplica esses dois conceitos da teoria de Goodman para destacar a diferença entre o pensamento discursivo e o não discursivo, isto é, entre um signo considerado como um elemento de um esquema de notação ou um contínuo estético. Quanto aos jogos de computador, essas categorias poderiam descrever experiências coincidentes, mas assíncronas. Em jogos mais contemplativos, como por exemplo The Journey (Thatgamecompany, 2012) e Shadow of the Colossus (Team ICO, 2005), os papéis do espectador e do jogador são borrados por uma temporalidade diferente que é mais propícia a turnos que dispensam qualquer necessidade de interação ou reação. Ademais, apesar do fundamento matemático dos jogos de computador, sempre existe a possibilidade de que um jogo também possa ser percebido analogicamente. E não apenas como uma apresentação para os espectadores - como Gadamer (1975, p. 104-105) já apontou, se um jogo é apresentado como uma representação para um público, torna-se um espetáculo (em alemão, schauspiel: literalmente, "jogo para ver") - mas também para os jogadores durante o jogo.
Portanto, os jogos de computador possibilitam uma experiência dessa dupla tensão entre os esquemas de representação analógicos e digitais.

Essa função de distanciamento também está relacionada às formas de interação, principalmente através da operação de um avatar. Vale ressaltar que este termo, como conceito para a representação gráfica de um usuário dentro de um espaço digital, surgiu como uma tentativa de fazer o jogador se sentir responsável pelos personagens no jogo Ultima IV: Quest of the Avatar (Origin Systems, 1985). A persona digital, como a origem hindu do termo sugere, deveria ser a encarnação do jogador no mundo virtual. Mas entre o jogador e o avatar há sempre uma distância causada pelo dispositivo que garante uma experiência segura. $O$ avatar nunca é o próprio jogador, mesmo ainda que assuma seu lugar no mundo do jogo. Ele atua como uma espécie de máscara. Por isso, o avatar não produz nem uma relação especular nem uma identidade. Já é algo diferente do jogador. Mesmo em jogos de primeira pessoa, sempre há uma perspectiva assíncrona e distópica que é percebida ou observada como um tipo de avatar. Existe uma distância persistente através do aparelho técnico e, especialmente, através da experiência desse fenômeno como jogo, como uma experiência de limiar. $\mathrm{O}$ avatar não é vivido, mas sim controlado, testado, em suma, jogado. Pode haver empatia, identificação, mas dificilmente constitui a experiência de ser outra pessoa.

Como dito anteriormente, já que o espaço de jogo digital é calculado e já dado, o jogador não precisa conhecer as regras para poder atuar nele, como seria o caso de um jogo não digital. Por essa razão, alguns pesquisadores argumentam pela impossibilidade, em jogos de computador, da existência de um "círculo mági- 
co" (Liebe, 2008) - ou seja, um outro mundo dentro do mundo, mas com regras frágeis e que pode ser interrompido a qualquer momento - ou mesmo da metacomunicação "isto é um jogo" (Neitzel, 2008). Além disso, os jogadores não podem quebrar ou alterar as regras durante o jogo, pois todas elas são pré-programadas.

A matematização do jogo, a sua capacidade contínua de repetição e a consequente falta de importância de estar ciente das regras implicam, pelo menos, duas consequências éticas. Em primeiro lugar, contrariamente ao teórico dos jogos de computador Jesper Juul (2013), que defende que os jogos de computador são um meio apropriado para experimentar o fracasso. A reversibilidade e a recuperabilidade do jogar digital, entretanto, impedem o sentimento de irreparabilidade, de perda e de trauma. Quando alguém perde um jogo constantemente, não há inexorável sentimento de fracasso, mas de frustração. O irreparável está fora do jogo. Se ele ocorrer, é sempre extrínseco ao jogo, já que, em sua maioria, o que é irreparável jaz na materialidade do jogo, ou seja, naquilo que escapa à sua lógica.

Em segundo lugar, a irrelevância gradual da metacomunicação "isto é um jogo" transforma o lúdico digital em um regime relacional que pode ser aplicado a outras formas de relações sociais mediadas, como redes sociais, plataformas de aprendizagem e aplicativos de namoro. Se, por um lado, este regime é eficaz para se alcançar metas e para aprender e melhorar habilidades, por outro lado, a incapacidade de se distinguir jogar de não jogar — de acordo com Bateson (1972, p. 197), também um sintoma de esquizofrenia — produz irresponsabilidade, ou melhor, não responsividade.

O jogo exige determinadas reações para que conti- nue. Mas interagir não é a mesma coisa que responder. A reversibilidade das ações e o caráter experimental impedem a singularidade do encontro com o radicalmente outro. A lógica digital leva experiência de limiar que é própria do jogo, ao seu extremo: não estar nem "dentro" nem "fora" para sempre reassegurar a possibilidade de retorno (sem nenhum "arranhão") para o interior, para o dentro de si, para o "mesmo". Já que a responsabilidade ética diante de um outro radical é uma consequência de uma experiência de alteridade, ela seria impossível de ocorrer apenas através de um jogo de computador. Em outras palavras, quando uma relação comunicacional assume uma estrutura de jogo, ela não denota o que uma relação comunicacional de fato denotaria, já que seria a própria experiência de alteridade.

Assim, quando se trata de comunicação por meios digitais, existe um risco persistente de que a comunicação se transforme em uma troca informacional e reativa, ou seja, quando a estrutura responsiva cede à lógica computacional do jogo. Ao perder a metacomunicação "isto é um jogo", o lúdico digital não é mais experienciado como um limiar. Perde-se a percepção dos contornos de sua moldura. Esse fato constitui o motor do potencial lúdico dos meios digitais e não é de se admirar que, nas últimas décadas, a estrutura de jogo digital tenha ultrapassado os limites dos jogos de computador, até o ponto em que a gamificação tenha se tornado um conceito importante para as redes sociais, resoluções de conflitos, programas de treinamento, processo educacional e mundo empresarial (Beil, 2013; Fuchs et al., 2014; Kapp, 2012).

No entanto, quando a comunicação é "jogada", ela perde suas características responsivas. $\mathrm{Na}$ "ilusão", não há um outro radical, mas sim jogadores ou objetos a serem jogados. O encontro em conjunto com os outros significa que o sujeito está sempre no controle. Por exemplo, a espacialidade segura do jogo regulado e reversível poderia conformar um discurso que não seria pronunciável em uma relação face a face ou diante de uma audiência (por exemplo, o discurso de ódio e difamação). Ademais, a estrutura de recompensa e a circularidade da lógica do jogo (por exemplo, botões "curtir", "compartilhar" e "recomendar") fornecem uma forma de comunicação desprovida de responsabilidade. Não é surpreendente que, quando um post criminal e ofensivo publicado em qualquer rede social recebe atenção da imprensa, a primeira coisa que o autor da publicação faz é tentar excluí-la, como se a estrutura de reversibilidade do jogo pudesse ser aplicada para a comunicação social. No entanto, não há um "botão de reset" na comunicação; apenas a possibilidade de reparação, depois de se ter assumido a responsabilidade.

Todas as interfaces de likes ou dislikes, muito comuns em aplicativos de namoro, se assemelham a estruturas de decisão simples dos jogos digitais. O perfil digital não representa uma pessoa, mas constitui um avatar que pode ser escolhido na esperança de um novo match ou varrido de uma vez por todas. Isso não tem nada a ver com o encontro de um rosto - como expressão e fonte de significado - , mas é a percepção de um objeto, de uma imagem que eu posso obliterar ou eliminar sem qualquer responsabilidade diante dela.

Entretanto, é necessário ressaltar que o argumento realizado até aqui não se apresenta como um diagnóstico pessimista do uso das tecnologias computacionais. Muito menos pretendo esboçar um gesto prescritivo, para dizer que essas ferramentas poderiam ser usadas de forma diferente. $\mathrm{O}$ objetivo destas breves reflexões 
é o de salientar que a lógica do jogo é inteiramente diferente da capacidade responsiva da comunicação. A falta de metacomunicação "isto é um jogo" é um dos motivos para o esquecimento de que a lógica por trás das mídias sociais não é a comunicação humana, mas o jogo digital matemático.

\section{Referências}

BATESON, Gregory. A Theory of Game and Fantasy. In: BATESON, Gregory. Steps to an Ecology of Mind. Northvale-NJ/London: Jason Aronson, 1972. p. 183-99.

BEIL, Benjamin. Game Studies: Eine Einführung. Berlin: Lit, 2013.

CAILLOIS, Roger. Man, Play, and Games. Trad. Meyer Barash. Chicago: University of Illinois Press, 2001.

FUCHS, Mathias; FIZEK, Sonia; RUFFINO, Paolo; SCHRA$P E$, Niklas (org.). Rethinking Gamification. Lüneburg: Meson Press by Hybrid, 2014.

GADAMER, Hans-Georg. Wahrheit und Methode: Grundzuge Der Philosophischen Hermeneutik. Tübingen: Mohr Siebeck, 1975

GOODMAN, Nelson. Languages of Art: An Approach to a Theory of Symbols. Indianapolis/New York/Kansas City: Hackett Publishing, 1968.

GÜNZEL, Stephan. The Mediality of Computer Games. In: FROMME, Johannes; UNGER, Alexander (org.). Computer Games and New Media Cultures. Dordrecht: Springer Netherlands, 2012. p. 31-46.
HOAD, T. F. Illusion. The Concise Oxford Dictionary of English Etymology. Oxford University Press, 1996.

HUIZINGA, Johan. Homo Ludens: A Study of the Play-Element in Culture. London: Routledge \& Kegan Paul, 1980.

JUUL, Jesper. The Art of Failure: An Essay on the Pain of Playing Video Games. Cambridge: The MIT Press, 2013.

KAPP, Karl M. The Gamification of Learning and Instruction: Game-Based Methods and Strategies for Training and Education. Hoboken: John Wiley \& Sons, 2012.

LIEBE, Michael. There Is No Magic Circle: On the Difference between Computer Games and Traditional Games. In: GÜNZEL, Stephan; LIEBE, Michael; MERSCH, Dieter (org.). Philosophy of Computer Games. Potsdam: Potsdam University Press, 2008. p. 324-41.

MAHRENHOLZ, Simone. Analogisches Denken. Aspekte Nicht-Diskursiver Rationalität. In: MERSCH, Dieter (org.). Die Medien der Künste: Beiträge zur Theorie des Darstellens. Munique/Paderborn: Wilhelm Fink, 2003. p. 75-92.

MERSCH, Dieter. Logik und Medialität des Computerspiels. Eine Medientheoretische Analyse. In: DISTELMAYER, Jan; HANKE, Christine; MERSCH, Dieter (org.). Game over!? Perspektiven Des Computerspiels. Bielefeld: transcript, 2008. p. 9-41.

Ordo Ab Chao: Order from Noise. Berlin/Züri- ch: Diaphanes, 2014.

NEITZEL, Britta. Metacommunicative Circles. In: GÜNZEL, Stephan; LIEBE, Michael; MERSCH, Dieter (org.). Philosophy of Computer Games. Potsdam: Potsdam University Press, 2008. p. 278-95.

PIAS, Claus. Computer Spiel Welten. Munique: Sequenzia, 2002.

RAUTZENBERG, Markus. Gerahmte Ungewissheit. Medialiät dnd Kontingenz im digitalen Zeitalter. WULF, Wulf; ZABOTKINA, Vera (org). Paragrana 24 (2), Berlin: De Gruyter, 2015. p. 57-73.

VON NEUMANN, John; MORGENSTERN, Oskar. Theory of Games and Economic Behavior. Princeton: Princeton University Press, 1944.

WOLF, Mark. The Medium of the Video Game. Austin: University of Texas Press, 2010.

\section{Referências audiovisuais}

NAUGHTY DOG (2013). The Last of Us. Los Angeles:

Sony Computer.

ORIGIN SYSTEMS (1985). Ultima IV: Quest of the Avatar. Origin Systems.

TEAM ICO (2005). Shadow of the Colossus. Los Angeles. Sony Computer Entertainment.

THATGAMECOMPANY. (2012). The Journey. Los Angeles: Sony Computer Entertainment. 


\section{Notas}

1 Doutor em Ciências da Comunicação pela USP e mestre em Comunicação e Cultura pela UFRJ. Atualmente, é professor colaborador do Programa de Pós-Graduação em Comunicação da UFPR, onde realiza estágio pós-doutoral com bolsa do Programa Nacional de Pós-Doutorado (PNPD/CAPES). E-mail: mauricioliesen@usp.br.

2 O lúdico e o jogo são concebidos neste trabalho para efetuar uma diferenciação conceitual presente no inglês entre os termos play e game.

3 Todas as traduções de citações de obras estrangeiras são de responsabilidade do autor deste texto.

4 O termo outridade é empregado neste texto em contraste ao conceito de alteridade. O primeiro refere-se a uma relação mais simples com "aquilo que não sou eu", podendo ser assimilado após um período inicial de estranhamento. Já a alteridade denota uma relação na qual o outro permanece sempre inacessível e inapreensível pelas categorias de si ou do "mesmo". 